\title{
Pharmacologic Thromboprophylaxis and Thrombosis in Hospitalized Patients with COVID-19: A Pooled Analysis
}

\author{
Rushad Patell ${ }^{1, *}$ Thita Chiasakul ${ }^{1,2, *}$ Ethan Bauer ${ }^{3}$ Jeffrey I. Zwicker ${ }^{1}$ \\ ${ }^{1}$ Division of Hemostasis and Thrombosis, Beth Israel Deaconess \\ Medical Center, Harvard Medical School, Boston, Massachusetts, \\ United States \\ 2 Division of Hematology, Department of Medicine, Faculty of \\ Medicine, Chulalongkorn University and King Chulalongkorn \\ Memorial Hospital, Thai Red Cross Society, Bangkok, Thailand \\ ${ }^{3}$ Sackler School of Medicine, Tel Aviv University, Tel Aviv, Israel

\begin{abstract}
Address for correspondence Jeffrey I. Zwicker, MD, Beth Israel Deaconess Medical Center, 330 Brookline Avenue, Boston, MA 02215 ,
\end{abstract} \\ United States (e-mail: jzwicker@bidmc.harvard.edu). \\ Thromb Haemost 2021;121:76-85.
}

\begin{abstract}
Keywords

- COVID-19

- anticoagulation

- thrombosis

- bleeding

Background Coronavirus disease 2019 (COVID-19) increases thrombosis in hospitalized patients prompting adoption of different thromboprophylaxis strategies. Safety and efficacy of escalated-dose pharmacologic thromboprophylaxis are not established. Objectives To determine the pooled incidence of thrombosis/bleeding in hospitalized patients with COVID-19 for standard-dose, intermediate-dose, therapeutic anticoagulation, and no pharmacologic thromboprophylaxis.

Methods MEDLINE, EMBASE, and Cochrane CENTRAL were searched up to August 29, 2020 for studies reporting pharmacologic thromboprophylaxis and thrombosis or bleeding. Pooled event rates were calculated using a random-effects model.

Results Thirty-five observational studies were included. The pooled incidence rates of total venous thromboembolism $(N=4,685)$ were: no prophylaxis $41.9 \%(95 \%$ confidence interval $\left.[\mathrm{Cl}]: 28.1-57.2, I^{2}=76 \%\right)$, standard-dose prophylaxis $19.8 \%(95 \% \mathrm{Cl}$ : 13.2-28.6, $\left.I^{2}=95 \%\right)$, intermediate-dose prophylaxis $11.9 \%(95 \% \mathrm{Cl}: 4.3-28.6$, $\left.I^{2}=91 \%\right)$, and therapeutic-dose anticoagulants $10.5 \%\left(95 \% \mathrm{Cl}: 4.2-23.8, I^{2}=82 \%\right.$, $p=0.003)$. The pooled incidence rates of arterial thrombosis $(N=1,464)$ were: no prophylaxis $11.3 \%$ (95\% Cl: $5.2-23.0, I^{2}=0 \%$ ), standard-dose prophylaxis $2.5 \%(95 \% \mathrm{Cl}$ : $\left.1.4-4.3, I^{2}=45 \%\right)$, intermediate-dose prophylaxis $2.1 \%\left(95 \% \mathrm{Cl}: 0.5-7.7, I^{2}=45 \%\right)$, and therapeutic-dose anticoagulants $1.3 \%\left(95 \% \mathrm{Cl}: 0.2-8.8, \mathrm{I}^{2}=0, p=0.009\right)$. The pooled bleeding event rates $(N=6,393)$ were nonsignificantly higher in therapeutic-dose anticoagulants compared with standard-dose prophylaxis, (6.3 vs. $1.7 \%, p=0.083$ ). Conclusion Thrombosis rates were lower in hospitalized COVID-19 patients who received pharmacologic thromboprophylaxis. Thrombosis and bleeding rates for patients receiving intermediate-dose thromboprophylaxis or therapeutic anticoagulation were similar to those who received standard-dose pharmacologic thromboprophylaxis.
\end{abstract}

These two authors contributed equally to this work.

received

September 22, 2020

accepted after revision

November 8, 2020 (c) 2021. Thieme. All rights reserved. Georg Thieme Verlag KG,

Rüdigerstraße 14,

70469 Stuttgart, Germany
DOI https://doi.org/ 10.1055/s-0040-1721664. ISSN 0340-6245. 


\section{Introduction}

The ongoing coronavirus disease 2019 (COVID-19) pandemic has affected over 20 million people globally since the emergence in December 2019 of severe acute respiratory syndrome coronavirus 2 (SARS-CoV-2) in China. ${ }^{1}$ It is now well recognized that patients with COVID-19 are predisposed to venous and arterial thromboses. ${ }^{2}$ Mechanisms linking the viral illness to the prothrombotic state are not fully elucidated but potential links include an immunothrombosis mediated through activated neutrophils and platelets, proinflammatory cytokine storm, complement activation, and endothelial injury. ${ }^{3-6}$

High rates of thrombosis in hospitalized patients with COVID-19 have been reported from centers across the world as the pandemic spread. ${ }^{7-11}$ This has led to varying pharmacologic thromboprophylaxis use in cohorts of patients with COVID-19 based on rapidly changing societal guidance, institutional protocols from local expertise, and geographic patterns of practice. ${ }^{12-18}$ Current national and international guidelines recommend universal pharmacologic thromboprophylaxis with subcutaneous low-molecular-weight heparin (LMWH) or unfractionated heparin in hospitalized patients. ${ }^{12,13,15,16,19-25}$ Institutional practices have varied based on individual and collective experience and range from standard prophylactic doses to full therapeutic anticoagulation strategies in select populations. ${ }^{26,27}$ Although randomized controlled studies evaluating different anticoagulation strategies are at various stages of development, there is no current consensus on best practices regarding the use of anticoagulation in this population to prevent thrombosis given the lack of high-quality prospective data.

Due to the current equipoise surrounding pharmacologic thromboprophylaxis in hospitalized patients with COVID-19 coupled with maturing observational data in the field, we conducted a systematic review and pooled analysis of studies reporting thrombotic (arterial or venous) events according to anticoagulation status. We compared summary thrombosis rates in hospitalized patients with COVID-19 stratified by anticoagulation dosing (none, standard dosage, intermediate dose, and full therapeutic anticoagulation) to assess the impact of anticoagulation on thrombosis and bleeding outcomes.

\section{Methods}

The study protocol is registered on PROSPERO (CRD42020203107). We followed the Preferred Reporting Items for Systematic Reviews and Meta-analyses (PRISMA) guidelines. The PRISMA 27-item checklist pertaining to the content of a systematic review and meta-analysis is presented in - Supplementary Table S1 (available in the online version).

\section{Data Sources and Search Strategies}

We searched MEDLINE, EMBASE, and Cochrane CENTRAL from inception to August 29, 2020. The following search terms were used: ("thrombosis" OR "thromboembolism" OR "acute coronary syndrome" OR "stroke") AND ("Novel coronavirus 2019” OR “COVID-19” OR "SARS-CoV-2” OR
“2019-nCoV"). No language restriction was applied. Reference lists of relevant studies and review articles were screened for potentially eligible studies.

\section{Study Selection}

Three authors (T.C., R.P., and E.B.) independently searched the literature, screened titles and abstracts, and reviewed full texts to identify potentially eligible studies. Disagreements were resolved by consensus or a fourth reviewer (J.I.Z.) when necessary. Eligible studies were randomized controlled trials, retrospective and prospective observational studies, or case series of adults (aged $\geq 18$ years) hospitalized with SARS-CoV-2 infection, which were confirmed by a standardized test or clinical criteria. Studies were required to report the pharmacologic thromboprophylaxis strategies that were used in the study cohort as well as the thrombosis rates and/or bleeding rates within each group. Studies were excluded if they were secondary publications (such as commentaries, editorials, and reviews), enrolled fewer than 10 patients, or published in language other than English. If multiple studies used the same or overlapping samples, we included only the one with the largest sample size in the quantitative analysis.

\section{Data Extraction}

Three authors (T.C., R.P., and E.B.) independently extracted data from included studies in duplicate using a standardized evidence table. Discrepancies were resolved by consensus or a fourth reviewer (J.I.Z.) when necessary. Pharmacologic thromboprophylaxis strategies were categorized into the following four groups according to the anticoagulation dosage: (1) No prophylaxis, (2) Standard-dose prophylaxis (enoxaparin $40 \mathrm{mg}$ per day or equivalent dosing of other anticoagulant including other LMWH, unfractionated heparin, or direct oral anticoagulant [DOAC]), (3) Intermediatedose prophylaxis (weight-adjusted, double-dose prophylaxis, or any dosage that is greater than the standard dose and lower than the therapeutic-dose anticoagulants), and (4) Therapeutic-dose anticoagulants (enoxaparin $1 \mathrm{mg} / \mathrm{kg}$ twice daily or $1.5 \mathrm{mg} / \mathrm{kg}$ once daily or equivalent doses of other anticoagulants including other LMWH, unfractionated heparin, or DOAC). The primary outcome was the rate of symptomatic or asymptomatic venous thromboembolism (VTE; lower and upper extremity deep vein thrombosis [DVT], pulmonary embolism, and catheter-associated thrombosis) in each pharmacologic thromboprophylaxis group. The secondary outcomes were the rates of arterial thrombosis (acute coronary syndrome and cerebrovascular accidents), bleeding, and overall mortality. Bleeding events were extracted as defined by individual studies. The following data were collected: authors, year of publication, country of study, study design, inclusion and exclusion criteria, baseline characteristics of participants, pharmacologic thromboprophylaxis strategies and regimen, number of participants in each group, methods and timing of VTE assessments, use of screening for asymptomatic DVT, definition of bleeding, and rates of the primary and secondary outcomes in each group. 


\section{Quality Assessment}

Methodological quality assessment of included studies was performed independently by three authors (T.C., R.P., and E.B.) using the validated methodological index for nonrandomized studies (MINORS) quality score at the study level. ${ }^{28}$ MINORS included eight and 12 methodological items for noncomparative studies and comparative studies, respectively. Each item was scored 0 if not reported; 1 when reported but inadequate; and 2 when reported and adequate. The global ideal score was 16 for noncomparative studies and 24 for comparative studies. Included studies were classified according to the overall MINORS score as having low, ${ }^{13-16}$ moderate, ${ }^{9-12}$ or high risk of bias ( $<9)$. Any differences in quality rating were resolved by consensus or adjudication by a fourth reviewer (J.I.Z.).

\section{Statistical Analysis}

Data analysis was performed using Comprehensive Metaanalysis (version 3.0. Eaglewood, New Jersey, United States). Pooled event rates of VTE, arterial thrombosis, and bleeding with $95 \%$ confidence intervals (CIs) were calculated by the DerSimonian and Laird method using a random-effects model. A Cochrane $Q$ test was used to assess the difference between pharmacologic thromboprophylaxis strategy groups. A $p$-value of $<0.05$ was considered significant for between-group heterogeneity. Interstudy heterogeneity was evaluated using the Cochran Q test and $I^{2}$ statistic. A Cochrane Q test $p$-value of $<0.05$ was considered significant for interstudy heterogeneity. An $I^{2}$ value of $\leq 25 \%$ represents insignificant heterogeneity, 26 to $50 \%$ low heterogeneity, 51 to $75 \%$ moderate heterogeneity, and $>75 \%$ high heterogeneity. Prespecified subgroup and sensitivity analyses were performed in patients requiring intensive care and in studies using screening ultrasonography to detect asymptomatic VTE. The presence of publication bias was assessed using funnel plots of event rates versus standard error and Egger's regression test, for which a $p$-value of $<0.1$ was considered significant for publication bias.

\section{Results}

\section{Study Identification}

The PRISMA flow diagram is shown in - Fig. 1. A total of 2,458 records were retrieved from the literature search. After screening by title and abstract, 2,372 records were excluded. The remaining 86 references underwent full-text review, 35 of which met eligibility criteria and were included in the analysis. These 35 studies collectively enrolled 10,857 patients diagnosed with SARS-CoV-2 infection. No additional eligible studies were identified by screening the reference
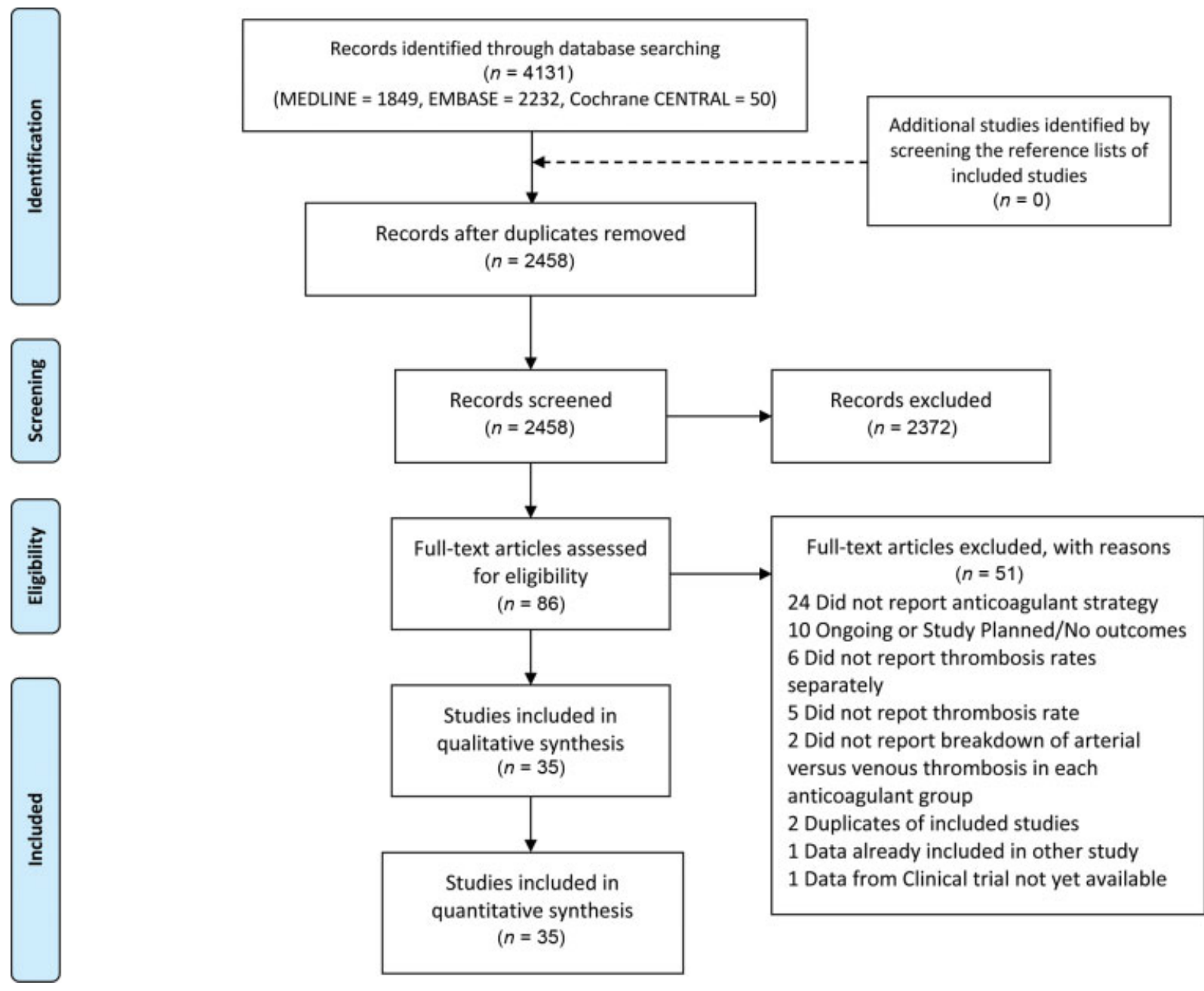

Fig. 1 PRISMA diagram. 
lists of included studies. A total of 4,685 patients were analyzed for VTE, 1,464 patients for arterial thrombosis, and 6,393 patients for bleeding outcomes. All 35 studies were observational studies. We did not identify any randomized controlled trial that met the eligibility criteria.

\section{Study Characteristics and Quality Appraisal}

The characteristics of included studies and the methodological quality assessment are summarized in - Supplementary Tables S1 and $\mathbf{S 2}$ (available in the online version).

Of the 35 observational studies included, four were prospective ${ }^{9,29-31}$ and the remaining 30 were cross-sectional and retrospective studies. ${ }^{7,11,30,32-58}$ All studies enrolled hospitalized patients with confirmed diagnosis of COVID-19; 11 focused on intensive care unit (ICU) population. $7,29,30,38,40,42,48,50-53$ The total numbers of studies reporting the incidence of VTE or bleeding in patients receiving no prophylaxis, standard-dose prophylaxis, intermediate-dose prophylaxis, and therapeuticdose anticoagulants were $7,32,8$, and 13 , respectively. Anticoagulants used for pharmacologic thromboprophylaxis included unfractionated heparins, LMWH, and DOACs. All studies were conducted during January and May 2020. Systematic screening for asymptomatic DVT was performed in 16 studies. $9,11,29,30,33,35-37,40,42,48-50,53,55,58$ Among the included studies, 6 studies reported bleeding events: 2 utilized the International Society of Thrombosis and Hemostasis bleeding criteria $^{56,59}$; 1 utilized the World Health Organization bleeding grading system ${ }^{32} ; 1$ defined clinically significant bleeding as any bleeding requiring or resulting in red cell transfusion, cessation of anticoagulation, or administration of reversal agents ${ }^{54}$; and 2 did not specify the definition for bleeding events. $^{35,57}$

For quality appraisal, 31 studies (89\%) scored 11 to 13 out of the 16 ideal global score for MINORS index. Scores were deducted from the items "unbiased assessment of the study endpoint" and "prospective calculation of the study size" in all studies. None of the included studies reported blinding of VTE assessors from COVID-19 status or pharmacologic thromboprophylaxis regimens. Eleven and 24 studies were classified as having low and moderate risk of bias, respectively. None of the studies included were classified as having high risk of bias.

\section{Pharmacologic Thromboprophylaxis Strategies and Incidence of VTE}

The pooled incidence of total VTE according to the pharmacologic thromboprophylaxis received were as follows: no prophylaxis $41.9 \%$ (95\% CI: $28.1-57.2, I^{2}=76 \%$ ), standard-dose prophylaxis $19.8 \%$ (95\% CI: $\left.13.2-28.6, I^{2}=95 \%\right)$, intermediatedose prophylaxis $11.9 \%$ (95\% CI: $4.3-28.6, I^{2}=91 \%$ ), and therapeutic-dose anticoagulants $10.5 \%$ (95\% CI: 4.2-23.8, $I^{2}=82 \%$ ) ( $p$-value for between-group heterogeneity $=0.003$; -Fig. 2 and -Table 1). Pair-wise comparison revealed a significant difference between no prophylaxis versus standard-dose prophylaxis ( $p=0.007)$, no prophylaxis versus intermediate-dose prophylaxis $(p=0.009$ ), and no prophylaxis versus therapeutic-dose anticoagulants $(p=0.002)$. Notably, there was no significant difference in the pair-wise compari- son between standard-dose prophylaxis versus intermediatedose prophylaxis $(p=0.32)$ or therapeutic-dose anticoagulants $(p=0.18$ ). Funnel plots (- Supplementary Fig. S1A, available in the online version) and Egger's regression asymmetry tests were performed to evaluate for publication bias. The funnel plot was symmetrical upon visual examination and there was no significant publication bias $(p=0.18)$.

\section{Pharmacologic Thromboprophylaxis Strategies and Incidence of Arterial Thrombosis}

The pooled incidence rates of arterial thrombosis according to the pharmacologic thromboprophylaxis received were as follows: no prophylaxis $11.3 \%$ (95\% CI: $5.2-23.0, I^{2}=0 \%$ ), standard-dose prophylaxis $2.5 \%$ (95\% CI: $\left.1.4-4.3, I^{2}=45 \%\right)$, intermediate-dose prophylaxis $2.1 \%$ (95\% CI: $0.5-7.7, I^{2}=45 \%$ ), and therapeutic-dose anticoagulants $1.3 \%\left(95 \% \mathrm{CI}: 0.2-8.8, I^{2}=0\right)$ ( $p$-value for between-group heterogeneity $=0.009$; - Table 1 and - Supplementary Fig. S2, available in the online version). Pair-wise comparison revealed significant difference between no prophylaxis versus standard-dose prophylaxis $(p=0.002)$, intermediate-dose prophylaxis $(p=0.03)$, and therapeuticdose anticoagulants $(p=0.04)$. There was no significant difference in the pair-wise comparison between standard-dose prophylaxis versus intermediate-dose prophylaxis $(p=0.81)$ or therapeutic-dose anticoagulants $(p=0.54)$. The funnel plot was symmetrical upon visual examination (-Supplementary Fig. S1B, available in the online version) and there was no significant publication bias $(p=0.50)$.

\section{Pharmacologic Thromboprophylaxis Strategies and Incidence of Bleeding}

Among the limited number of studies where data were available, bleeding events were numerically higher in therapeutic-dose anticoagulants compared with standard-dose prophylaxis (6.3 vs. 1.7\%; - Table 1 and -Supplementary Fig. $\mathbf{5 3}$ [available in the online version]). The funnel plot was symmetrical upon visual examination (-Supplementary Fig. S1C, available in the online version) and there was no significant publication bias $(p=0.61)$.

\section{Pharmacologic Thromboprophylaxis Strategies and Overall Mortality}

The pooled rates of overall mortality were $23.1 \%$ (95\% CI: $4.3-67.1, I^{2}=96 \%$ ) in the no prophylaxis group and $21.2 \%$ $\left(95 \% \mathrm{CI}: 17.3-25.7, I^{2}=57 \%\right)$ in the standard-dose prophylaxis group. There was one study that reported overall mortality rate in the intermediate-dose prophylaxis group $(21.0 \%, 95 \% \mathrm{CI}$ : 14.2-29.8), and one study in therapeutic-dose anticoagulants group (16.8\%, 95\% CI: 15.0-18.8). There was no significant difference among the pharmacologic thromboprophylaxis strategies ( $p$-value for between-group heterogeneity $=0.19$; -Table 1 and - Supplementary Fig. 54 [available in the online version]).

\section{Subgroup and Sensitivity Analyses}

A prespecified subgroup analysis was performed in only patients in the ICU (-Supplementary Table S4, available in the online version). There was no significant difference in the 
80 Thromboprophylaxis and Thrombosis in Hospitalized Patients with COVID-19 Patell et al.

Study name

Alonso-Fernandez et al. 2020

Cho et al. 2020

Cui et al. 2020

Grandmaison et al. 2020

Lodigiani et al 2020

Trimaille et al. 2020

Zhang et al. 2020

Aleva et al. 2020

Alonso-Fernandez et al. 2020

Al-Samkari et al. 2020

Artifoni et al. 2020

Bompard et al. 2020

Chen et al. 2020

Cho et al. 2020

Criel et al. 2020

Demelo-Rodriguez et al. 2020

Dugar et al. 2020

Faulvel et al. 2020

Grandmaison et al. 2020

Klok et al. 2020

Koleilat et al, 2020

Le Jeune et al. 2020

Litjos et al. 2020

Lodigiani et al 2020

Longchamp et al. 2020

Mei et al. 2020

Middeldorp et al. 2020

Moll et al. 2020

Nahum et al. 2020

Pesavento et al. 2020

Pizzolo et al. 2020

Ren et al. 2020

Santoliquido et al. 2020

Tavazzi et al. 2020

Trimaille et al. 2020

Zhang et al. 2020

Bompard et al. 2020

Criel et al. 2020

Faulvel et al. 2020

Le Jeune et al. 2020

Lodigiani et al 2020

Mattioli et al. 2020

Middeldorp et al. 2020

Trimaille et al. 2020

Klok et al. 2020

Koleilat et al, 2020

Litjos et al. 2020

Lodigiani et al 2020

Longchamp et al. 2020

Moll et al. 2020

Pesavento et al. 2020

Thomas et al. 2020

Trimaille et al. 2020

\begin{tabular}{|c|c|c|c|}
\hline $\begin{array}{c}\text { Event } \\
\text { rate }\end{array}$ & $\begin{array}{c}\text { Lower } \\
\text { limit }\end{array}$ & $\begin{array}{c}\text { Upper } \\
\text { limit }\end{array}$ & Total \\
\hline 0.750 & 0.238 & 0.966 & $3 / 4$ \\
\hline 0.357 & 0.157 & 0.624 & $5 / 14$ \\
\hline 0.247 & 0.165 & 0.352 & $20 / 81$ \\
\hline 0.833 & 0.194 & 0.990 & $2 / 2$ \\
\hline 0.245 & 0.148 & 0.378 & $13 / 53$ \\
\hline 0.613 & 0.435 & 0.765 & $19 / 31$ \\
\hline 0.489 & 0.388 & 0.591 & $44 / 90$ \\
\hline 0.419 & 0.281 & 0.572 & \\
\hline 0.360 & 0.240 & 0.501 & $18 / 50$ \\
\hline 0.462 & 0.284 & 0.650 & $12 / 26$ \\
\hline 0.054 & 0.034 & 0.083 & $19 / 354$ \\
\hline 0.225 & 0.143 & 0.337 & $16 / 71$ \\
\hline 0.180 & 0.119 & 0.263 & $20 / 111$ \\
\hline 0.455 & 0.354 & 0.559 & $40 / 88$ \\
\hline 0.326 & 0.255 & 0.407 & $47 / 144$ \\
\hline 0.041 & 0.010 & 0.149 & $2 / 49$ \\
\hline 0.147 & 0.100 & 0.212 & $23 / 156$ \\
\hline 0.389 & 0.198 & 0.621 & $7 / 18$ \\
\hline 0.024 & 0.015 & 0.038 & $18 / 738$ \\
\hline 0.538 & 0.350 & 0.716 & $14 / 26$ \\
\hline 0.389 & 0.318 & 0.465 & $65 / 167$ \\
\hline 0.140 & 0.081 & 0.230 & $12 / 86$ \\
\hline 0.160 & 0.061 & 0.357 & $4 / 25$ \\
\hline 0.944 & 0.495 & 0.997 & $8 / 8$ \\
\hline 0.040 & 0.019 & 0.082 & $7 / 175$ \\
\hline 0.348 & 0.184 & 0.557 & $8 / 23$ \\
\hline 0.020 & 0.008 & 0.046 & $5 / 256$ \\
\hline 0.033 & 0.012 & 0.083 & $4 / 123$ \\
\hline 0.047 & 0.024 & 0.092 & $8 / 169$ \\
\hline 0.794 & 0.627 & 0.899 & $27 / 34$ \\
\hline 0.025 & 0.011 & 0.055 & $6 / 240$ \\
\hline 0.279 & 0.166 & 0.430 & $12 / 43$ \\
\hline 0.854 & 0.724 & 0.929 & $41 / 48$ \\
\hline 0.119 & 0.065 & 0.207 & $10 / 84$ \\
\hline 0.204 & 0.117 & 0.332 & $11 / 54$ \\
\hline 0.112 & 0.072 & 0.169 & $19 / 170$ \\
\hline 0.415 & 0.291 & 0.551 & $22 / 53$ \\
\hline 0.198 & 0.132 & 0.286 & \\
\hline 0.500 & 0.310 & 0.690 & $12 / 24$ \\
\hline 0.133 & 0.051 & 0.306 & $4 / 30$ \\
\hline 0.051 & 0.021 & 0.116 & $5 / 99$ \\
\hline 0.100 & 0.014 & 0.467 & $1 / 10$ \\
\hline 0.071 & 0.032 & 0.150 & $6 / 84$ \\
\hline 0.010 & 0.001 & 0.064 & $1 / 105$ \\
\hline 0.467 & 0.357 & 0.579 & $35 / 75$ \\
\hline 0.065 & 0.016 & 0.224 & $2 / 31$ \\
\hline 0.119 & 0.043 & 0.286 & \\
\hline 0.176 & 0.058 & 0.427 & $3 / 17$ \\
\hline 0.087 & 0.022 & 0.289 & $2 / 23$ \\
\hline 0.556 & 0.330 & 0.760 & $10 / 18$ \\
\hline 0.013 & 0.002 & 0.088 & $1 / 76$ \\
\hline 0.167 & 0.010 & 0.806 & $0 / 2$ \\
\hline 0.048 & 0.007 & 0.271 & $1 / 21$ \\
\hline 0.036 & 0.012 & 0.105 & $3 / 84$ \\
\hline 0.270 & 0.175 & 0.392 & $17 / 63$ \\
\hline 0.035 & 0.009 & 0.130 & $2 / 57$ \\
\hline 0.105 & 0.042 & 0.238 & \\
\hline
\end{tabular}

Event rate and $95 \% \mathrm{Cl}$

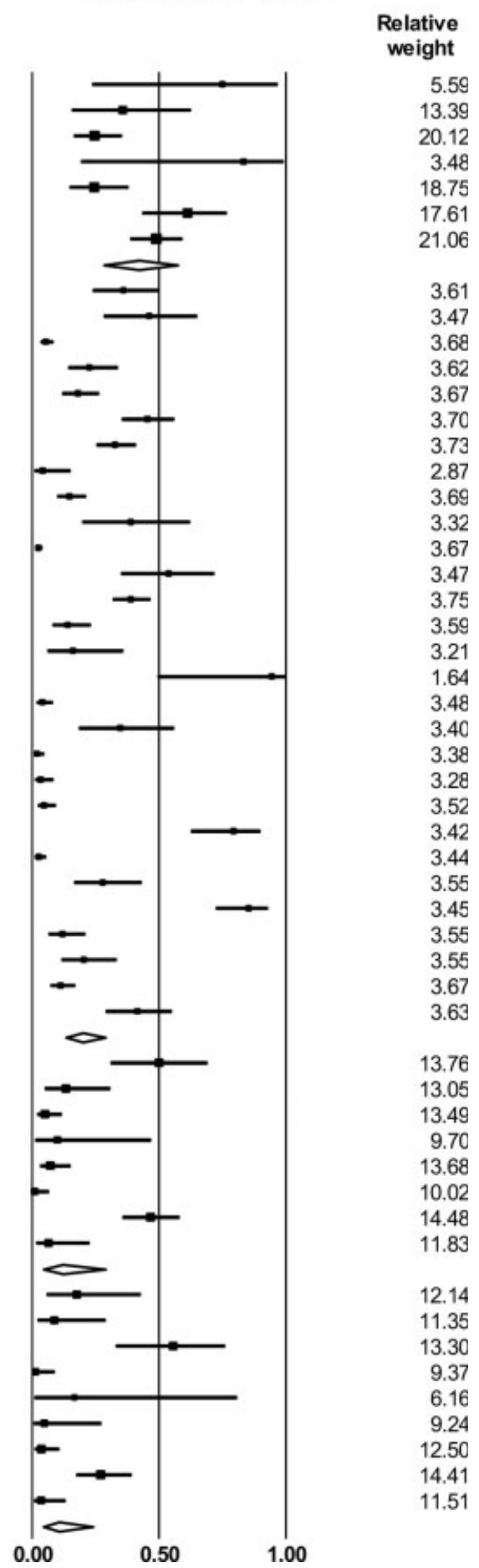

Fig. 2 Forest plot showing the pooled incidence of total venous thromboembolism amongst pharmacologic thromboprophylaxis strategies: no prophylaxis, standard prophylaxis, intermediate prophylaxis, and full-dose therapeutic anticoagulation. Total VTE included symptomatic or asymptomatic VTE (lower and upper extremity DVT, PE, and catheter-associated thrombosis). DVT, deep vein thrombosis; PE, pulmonary embolism; VTE, venous thromboembolism.

total VTE rate among the pharmacologic thromboprophylaxis strategy groups. Of note, the pooled incidence of total VTE was markedly higher in the ICU population (30.7-45.5\%) compared with the non-ICU counterpart (3.0-7.3\%), regardless of pharmacologic thromboprophylaxis strategies received. Similarly, in a sensitivity analysis that included only studies that performed a systematic screening for DVT by ultrasound, there was no significant difference in the total VTE rate among the pharmacologic thromboprophylaxis strategy groups (-Supplementary Table S4, available in the online version). Additional sensitivity analysis included studies that were judged to have a low risk of bias (based on a MINORS score of 13-16) and studies that explicitly stated that only thrombotic outcomes based on imaging studies 
Table 1 Comparison of total venous thromboembolism, deep vein thrombosis, pulmonary embolism, arterial thrombosis, bleeding events, and mortality according to pharmacologic thromboprophylaxis strategies

\begin{tabular}{|c|c|c|c|c|c|c|}
\hline \multicolumn{2}{|l|}{ Outcomes } & \multirow{2}{*}{$\begin{array}{l}\text { No prophylaxis } \\
276(7)\end{array}$} & \multirow{2}{*}{$\begin{array}{l}\begin{array}{l}\text { Standard-dose } \\
\text { prophylaxis }\end{array} \\
3,589(29)\end{array}$} & \multirow{2}{*}{$\begin{array}{l}\begin{array}{l}\text { Intermediate-dose } \\
\text { prophylaxis }\end{array} \\
458(8)\end{array}$} & \multirow{2}{*}{$\begin{array}{l}\begin{array}{l}\text { Therapeutic } \\
\text { anticoagulants }\end{array} \\
362(9)\end{array}$} & \multirow[t]{2}{*}{$\begin{array}{l}\text { Overall } \\
p \text {-value }\end{array}$} \\
\hline Total VTE & $\begin{array}{l}\text { No. of participants } \\
\text { (No. of studies) }\end{array}$ & & & & & \\
\hline & $\begin{array}{l}\text { Pooled incidence, } \\
\%(95 \% \mathrm{Cl})\end{array}$ & $41.9(28.1-57.2)$ & $19.8(13.2-28.6)$ & $11.9(4.3-28.6)$ & $10.5(4.2-23.8)$ & 0.003 \\
\hline & $P^{2}, \%$ & 76 & 95 & 90 & 82 & \\
\hline \multirow[t]{3}{*}{ DVT } & $\begin{array}{l}\text { No. of participants } \\
\text { (No. of studies) }\end{array}$ & $148(3)$ & $1,816(18)$ & $189(3)$ & $125(3)$ & \\
\hline & $\begin{array}{l}\text { Pooled incidence, } \\
\%(95 \% \mathrm{Cl})\end{array}$ & $20.0(8.7-39.5)$ & $15.5(11.9-14.4)$ & $11.9(2.7-40.1)$ & $14.4(1.6-63)$ & 0.92 \\
\hline & $1^{2}, \%$ & 73 & 94 & 88 & 92 & \\
\hline \multirow[t]{3}{*}{$\mathrm{PE}$} & $\begin{array}{l}\text { No. of participants } \\
\text { (No. of studies) }\end{array}$ & $57(2)$ & $2,396(14)$ & $387(5)$ & $205(5)$ & \\
\hline & $\begin{array}{l}\text { Pooled incidence, } \\
\%(95 \% \mathrm{Cl})\end{array}$ & $29.9(1.2-93.5)$ & $6.5(2.9-14.1)$ & $7.3(1.8-25.4)$ & $11.2(4.3-26.4)$ & 0.68 \\
\hline & $1^{2}, \%$ & 88 & 95 & 90 & 69 & \\
\hline \multirow[t]{3}{*}{$\begin{array}{l}\text { Arterial } \\
\text { thrombosis }\end{array}$} & $\begin{array}{l}\text { No. of participants } \\
\text { (No. of studies) }\end{array}$ & $53(1)$ & $1,057(4)$ & $278(3)$ & $76(1)$ & \\
\hline & $\begin{array}{l}\text { Pooled incidence, } \\
\%(95 \% \mathrm{Cl})\end{array}$ & $11.3(5.2-23.0)$ & $2.5(1.4-4.3)$ & $2.1(0.5-7.7)$ & $1.3(0.2-8.8)$ & 0.009 \\
\hline & $I^{2}, \%$ & 0 & 45 & 45 & 0 & \\
\hline \multirow[t]{3}{*}{ Bleeding $^{d}$} & $\begin{array}{l}\text { No. of studies } \\
\text { (No. of participants) }\end{array}$ & $936(3)$ & $3,484(5)$ & $194(2)$ & $1,779(3)$ & \\
\hline & $\begin{array}{l}\text { Pooled incidence, } \\
\%(95 \% \mathrm{Cl})\end{array}$ & $6.7(2.2-19.0)$ & $1.7(1.0-2.9)$ & $2.1(0.8-5.4)$ & $6.3(1.5-22.7)$ & 0.08 \\
\hline & $I^{2}, \%$ & 71 & 65 & 0 & 96 & \\
\hline \multirow[t]{3}{*}{$\begin{array}{l}\text { Overall } \\
\text { mortality }\end{array}$} & $\begin{array}{l}\text { No. of participants } \\
\text { (No. of studies) }\end{array}$ & $981(2)$ & $2,529(5)$ & $105(1)$ & $1,530(1)$ & \\
\hline & $\begin{array}{l}\text { Pooled incidence, } \\
\%(95 \% \mathrm{Cl})\end{array}$ & $23.1(4.3-67.1)$ & $21.2(17.3-25.7)$ & $21.0(14.2-29.8)$ & $16.8(15.0-18.8)$ & 0.19 \\
\hline & $I^{2}, \%$ & 96 & 57 & 0 & 0 & \\
\hline
\end{tabular}

Abbreviations: $\mathrm{Cl}$, confidence intervals; DVT, deep vein thrombosis; PE, pulmonary embolism; VTE, venous thromboembolism.

${ }^{a} p$-Values were derived from the Q-test for heterogeneity among the four pharmacologic thromboprophylaxis strategy groups. A $p$-value of $<0.05$ was considered significant for between-group heterogeneity.

${ }^{\text {b} T o t a l ~ V T E ~ i n c l u d e d ~ s y m p t o m a t i c ~ o r ~ a s y m p t o m a t i c ~ V T E ~(l o w e r ~ a n d ~ u p p e r ~ e x t r e m i t y ~ D V T, ~ P E, ~ a n d ~ c a t h e t e r-a s s o c i a t e d ~ t h r o m b o s i s) . ~}$

'Arterial thrombosis included acute coronary syndrome and cerebrovascular accidents.

${ }^{\mathrm{d}}$ Bleeding events were extracted as defined by individual studies.

were included and had similar results (-Supplementary

Table S4, available in the online version). Notably several of these sensitivity analyses had limited numbers in the various categories limiting conclusions.

To further explore the source of heterogeneity, we performed sensitivity analyses according to the studies' geographic location (Europe, North America, and China; - Supplementary Fig. S5 and - Supplementary Table S5, available in the online version). However, high statistical heterogeneity persisted even within regions.

\section{Discussion}

In this pooled analysis that included 35 studies, we observed that the pooled incidence rates of VTE that were approximately $50 \%$ lower in patients receiving standard-dose pharmacologic thromboprophylaxis than in those who did not receive pharmacologic thromboprophylaxis. Compared with standard-dose prophylaxis, both intermediate and therapeutic anticoagulation were associated with lower pooled VTE rates and higher pooled bleeding rates, although the differences did not reach statistical significance. In a recently published systematic review of prophylactic anticoagulants in hospitalized patients with COVID-19, which only included studies that compared pharmacologic thromboprophylaxis with an active comparator, placebo, or no treatment, seven retrospective nonrandomized studies (5,929 participants) were identified. The reduction of all-cause mortality with prophylactic anticoagulants compared with no prophylaxis was inconsistent among the included studies and the analyses of VTE events were not performed due to the lack of data. ${ }^{60}$ Using a pooled analysis, our study quantifies and compares the estimates of these outcomes 
according to the dosage of pharmacologic thromboprophylaxis, and therefore, adding value to the existing literature.

This pooled analysis suggests that standard pharmacologic thromboprophylaxis is effective in preventing total venous thrombotic events during hospitalization compared with no anticoagulation and supports current societal guidance that recommends at least a universal pharmacologic thromboprophylaxis for all hospitalized patients with COVID-19. ${ }^{13-16,20}$ SARS-COV-2 infection is also associated with an increased risk of arterial thrombotic events including ischemic strokes. ${ }^{61} \mathrm{We}$ found that compared with patients who received no pharmacologic thromboprophylaxis, rates of arterial events were significantly lower in patients treated with any form of anticoagulant therapy, suggesting that the potential benefit of pharmacologic thromboprophylaxis may extend beyond that of preventing VTE.

Given the high rates of thrombosis despite standard-dose pharmacologic thromboprophylaxis, numerous institutions have operationalized protocols that include dose escalation to intermediate dosing (including weight-based or twicedaily dosing of LMWH) or therapeutic anticoagulation. ${ }^{27}$ Considerable practice variation exists regarding the indication for dose escalation. ${ }^{62}$ ICU admission, obesity, presence of additional VTE risk factors, and high D-dimer are among the reasons that lead physicians to escalate to therapeutic-dose anticoagulation. ${ }^{62}$ In our pooled analysis, we included eight studies with a total of 458 patients who received intermediate-dose prophylaxis. In such studies, the pooled total VTE trended lower than that of the 28 studies that reported on standard pharmacologic thromboprophylaxis (11.9 vs. 19.6\%) but was not statistically significant. Interestingly, the pooled rates of VTE reported for intermediate versus therapeutic dosing were similar, suggesting that there may be little additive benefit beyond a threshold dose of pharmacologic thromboprophylaxis.

Recent reports suggest high rates of hemorrhage among hospitalized patients with COVID-19. ${ }^{10}$ We noted a high rate of hemorrhage among the nonanticoagulant group, which potentially is a reflection of baseline hemorrhagic risk that influenced the decision to not administer pharmacologic thromboprophylaxis. Other explanations may include the difference in health care settings and the bleeding definitions and grading systems that were used in each study. The overall incidence of major hemorrhage among patients receiving therapeutic heparin was greater than $6 \%$, which is approximately fivefold higher than what is typically reported in primary pharmacologic thromboprophylaxis trials in hospitalized patients ${ }^{63-65}$ and approximately threefold higher than the pooled rates for standard and intermediate-dose prophylaxis in this analysis.

Critically ill patients are at increased risk of thrombosis compared with other hospitalized patients, at least in part from a constellation of prothrombotic risk factors such as prolonged immobilization and hyperinflammatory states. ${ }^{66}$ Among hospitalized patients with COVID-19, thrombosis rates are significantly higher in patients requiring critical care compared with ward-level care within a general medical area. ${ }^{67}$ In theory, a dosage higher than the standard anti- coagulation dosage for pharmacologic thromboprophylaxis would have the greatest impact among critically ill patients. However, in a subgroup analysis limited to ICU patients, none of the strategies were associated with preventing total venous thrombotic events.

\section{Limitations}

There are several limitations to this study, some of which are intrinsic to observational and retrospective cohort studies that were included which are prone to biases by design. Due to the lack of randomization, selection bias may result from the potential imbalances in the baseline characteristics of patients receiving different pharmacologic thromboprophylaxis regimens. Reports spanned the globe which may be beneficial in terms of generalizability but also likely contributed to high statistical heterogeneity. Other factors contributing to statistical heterogeneity included diverse populations, methods of COVID-19 diagnosis (standardized test vs. clinical criteria), severity of disease, institutional practices, and outcome measurement (such as definition of primary outcomes, inclusion of only symptomatic vs. asymptomatic events detected by systematic screening in some reports, and lack of blinding to treatments received, given majority of diagnoses were made in the clinical setting and duration of hospitalization). Several sensitivity subgroup analyses were performed to address the heterogeneity observed and the differences in outcomes' definitions, which are included in - Supplementary Tables $\mathbf{5 4}$ and S5 (available in the online version). Some of the groups are small, which limits interpretation but overall trends are consistent with the main analysis. Although anticoagulation strategies were grouped as standard, intermediate, and therapeutic dosing levels, each category included different therapeutic agents and varying algorithms. For instance, intermediatedose LMWH included both weight-based subtherapeutic strategies and twice-daily fixed dosing. The lack of granularity in data available and inadequate numbers do not permit comparing these strategies separately in a meaningful manner. Finally, we acknowledge that the subgroups are unbalanced, which may lead to challenges in statistical comparison. Thus, despite inclusion of approximately 800 patients receiving intermediate or therapeutic dosing regimens, insufficient power (compounded by interstudy heterogeneity) precludes definitive conclusions regarding relative efficacy or safety differences between these two approaches.

\section{Conclusion}

This pooled analysis showed that hospitalized patients with COVID-19 treated with standard-dose pharmacologic thromboprophylaxis have lower rates of thrombosis compared with those receiving no pharmacologic thromboprophylaxis, and supports current societal guideline recommendations. In this analysis, higher dosing regimens of pharmacologic thromboprophylaxis (i.e., intermediate or therapeutic dosage) were not associated with lower in-hospital thrombotic events compared with standard-dose prophylaxis. This is especially important considering the safety profile of intermediate-dose prophylaxis and therapeutic anticoagulation relative to standard-dose 
prophylaxis in hospitalized patients with COVID-19 is also not established. Multiple clinical trials are at different stages of development to examine the role of escalated doses of pharmacologic thromboprophylaxis in patients with COVID-19 (NCT04359277, NCT04345848, and NCT04344756). Until randomized and prospective data are available, this study provides estimates of benefits and risks with anticoagulation that can inform shared decision making with patients and practice decisions.

\section{What is known about this topic?}

- Novel coronavirus disease 2019 is associated with increased rates of venous and arterial thromboses in patients hospitalized with acute illness.

- Efficacy and safety of therapeutic or intermediate dosing of in-hospital thromboprophylaxis relative to standard dosing are not established.

\section{What does this paper add?}

- Rates of thrombosis are lower in hospitalized patients with COVID-19 receiving pharmacologic thromboprophylaxis compared with those who did not receive any anticoagulant treatment.

- Rates of thrombosis and bleeding for patients receiving intermediate-dose pharmacologic thromboprophylaxis or therapeutic anticoagulation were similar to those treated with standard-dose pharmacologic thromboprophylaxis.

- Data from randomized controlled trials are needed to determine the relative efficacy and safety profiles of the individual strategies for pharmacologic thromboprophylaxis.

\section{Funding}

The authors received no specific funding for this work.

\section{Conflict of Interest}

J.I.Z. reports research funding from Incyte and Quercegen; consultancy for Sanofi, CSL, and Parexel; and having been a member of honoraria/advisory boards of Pfizer/BMS, Portola, and Daiichi. R.P., T.C., and E.B. have no disclosures.

\section{References}

1 Medicine JHUa Coronavirus Resource Center Accessed May 16, 2020 at: https://coronavirus.jhu.edu/map.html

2 Connors JM, Levy JH. COVID-19 and its implications for thrombosis and anticoagulation. Blood 2020;135(23):2033-2040

3 Varga Z, Flammer AJ, Steiger P, et al. Endothelial cell infection and endotheliitis in COVID-19. Lancet 2020;395(10234):1417-1418

4 Iba T, Levy JH, Levi M, Thachil J. Coagulopathy in COVID-19. J Thromb Haemost 2020;18(09):2103-2109

5 Nicolai L, Leunig A, Brambs S, et al. Immunothrombotic dysregulation in COVID-19 pneumonia is associated with respiratory failure and coagulopathy. Circulation 2020;142(12):1176-1189
6 Violi F, Pastori D, Cangemi R, Pignatelli P, Loffredo L. Hypercoagulation and antithrombotic treatment in coronavirus 2019: a new challenge. Thromb Haemost 2020;120(06): 949-956

7 Klok FA, Kruip MJHA, van der Meer NJM, et al. Confirmation of the high cumulative incidence of thrombotic complications in critically ill ICU patients with COVID-19: An updated analysis. Thromb Res 2020;191:148-150

8 Oxley TJ, Mocco J, Majidi S, et al. Large-vessel stroke as a presenting feature of Covid-19 in the young. $\mathrm{N}$ Engl J Med 2020;382(20):e60

9 Demelo-Rodríguez P, Cervilla-Muñoz E, Ordieres-Ortega L, et al. Incidence of asymptomatic deep vein thrombosis in patients with COVID-19 pneumonia and elevated D-dimer levels. Thromb Res 2020;192:23-26

10 Al-Samkari H, Karp Leaf RS, Dzik WH, et al. COVID and coagulation: bleeding and thrombotic manifestations of SARS-CoV2 infection. Blood 2020;136(04):489-500

11 Zhang L, Feng X, Zhang D, et al. Deep vein thrombosis in hospitalized patients with coronavirus disease 2019 (COVID-19) in Wuhan, China: prevalence, risk factors, and outcome. Circulation 2020;142(02):114-128

12 Vivas D, Roldan V, Esteve-Pastor MA, et al. Recommendations on antithrombotic treatment during the COVID-19 pandemic. Position statement of the Working Group on Cardiovascular Thrombosis of the Spanish Society of Cardiology [in Spanish]. Rev Esp Cardiol 2020;73(09):749-757

13 Bikdeli B, Madhavan MV, Jimenez D, et al;Global COVID-19 Thrombosis Collaborative Group, Endorsed by the ISTH, NATF, ESVM, and the IUA, Supported by the ESC Working Group on Pulmonary Circulation and Right Ventricular Function. COVID-19 and thrombotic or thromboembolic disease: implications for prevention, antithrombotic therapy, and follow-up: JACC stateof-the-art review. J Am Coll Cardiol 2020;75(23):2950-2973

14 American Society of Hematology. COVID-19 and VTE/anticoagulation: frequently asked questions. Accessed May 19, 2020 at: http:// www.hematology.org/covid-19/covid-19-and-coagulopathy

15 Thachil J, Tang N, Gando S, et al. ISTH interim guidance on recognition and management of coagulopathy in COVID-19. J Thromb Haemost 2020;18(05):1023-1026

16 World Health Organization. Clinical management of severe acute respiratory infection (SARI) when COVID-19 disease is suspected: interim guidance. Accessed October 14, 2020 at: https://www. who.int/docs/default-source/coronaviruse/clinical-management -of-novel-cov.pdf

17 Cattaneo M, Bertinato EM, Birocchi S, et al. Pulmonary embolism or pulmonary thrombosis in COVID-19? Is the recommendation to use high-dose heparin for thromboprophylaxis justified?. Thromb Haemost 2020;120(08):1230-1232

18 Zhai Z, Li C, Chen Y, et al;Prevention Treatment of VTE Associated with COVID-19 Infection Consensus Statement Group. Prevention and treatment of venous thromboembolism associated with coronavirus disease 2019 infection: a consensus statement before guidelines. Thromb Haemost 2020;120(06):937-948

19 Taskforce NC-CE. Australian guidelines for the clinical care of people with COVID-19. Accessed October 13, 2020 at: http:// www.clinicalguidelines.gov.au/register/australian-guidelinesclinical-care-people-covid-19

20 Health NIo. COVID-19 Treatment Guidelines Panel. Coronavirus disease 2019 (COVID-19) treatment guidelines. Accessed October 13, 2020 at: http://www.covid19treatmentguidelines.nih.gov/

21 Kosior DA, Undas A, Kopeć G, et al. Guidance for anticoagulation management in venous thromboembolism during the coronavirus disease 2019 pandemic in Poland: an expert opinion of the Section on Pulmonary Circulation of the Polish Cardiac Society. Kardiol Pol 2020;78(06):642-646

22 Llau JV, Ferrandis R, Sierra P, et al. SEDAR-SEMICYUC consensus recommendations on the management of haemostasis disorders 
in severely ill patients with COVID-19 infection. Rev Esp Anestesiol Reanim 2020;67(07):391-399

23 Falavigna M, Colpani V, Stein C, et al. Guidelines for the pharmacological treatment of COVID-19. The task-force/consensus guideline of the Brazilian Association of Intensive Care Medicine, the Brazilian Society of Infectious Diseases and the Brazilian Society of Pulmonology and Tisiology. Rev Bras Ter Intensiva 2020;32 (02):166-196

24 Gerotziafas GT, Catalano M, Colgan MP, et al;Scientific Reviewer Committee. Guidance for the management of patients with vascular disease or cardiovascular risk factors and COVID-19: position paper from VAS-European Independent Foundation in Angiology/Vascular Medicine. Thromb Haemost 2020;120(12): 1597-1628

25 American Society of Hematology. COVID-19 and coagulopathy: frequently asked questions. Accessed May 19, 2020 at: http:// www.hematology.org/covid-19/covid-19-and-coagulopathy

26 Cohoon KP, Mahé G, Tafur AJ, Spyropoulos AC. Emergence of institutional antithrombotic protocols for coronavirus 2019. Res Pract Thromb Haemost 2020;4(04):510-517

27 Patell R, Midha S, Kimani S, et al. Variability in institutional guidance for COVID-19-associated coagulopathy in the United States. Thromb Haemost 2020;120(12):1725-1732

28 Slim K, Nini E, Forestier D, Kwiatkowski F, Panis Y, Chipponi J. Methodological index for non-randomized studies (minors): development and validation of a new instrument. ANZ J Surg 2003;73(09):712-716

29 Longchamp A, Longchamp J, Manzocchi-Besson S, et al. Venous thromboembolism in critically Ill patients with COVID-19: results of a screening study for deep vein thrombosis. Res Pract Thromb Haemost 2020;4(05):842-847

30 Nahum J, Morichau-Beauchant T, Daviaud F, et al. Venous thrombosis among critically ill patients with coronavirus disease 2019 (COVID-19). JAMA Netw Open 2020;3(05):e2010478

31 Alonso-Fernández A, Toledo-Pons N, Cosío BG, et al. Prevalence of pulmonary embolism in patients with COVID-19 pneumonia and high D-dimer values: a prospective study. PLoS One 2020;15(08): e0238216

32 Al-Samkari H, Karp Leaf RS, Dzik WH, et al. COVID-19 and coagulation: bleeding and thrombotic manifestations of SARSCoV-2 infection. Blood 2020;136(04):489-500

33 Artifoni M, Danic G, Gautier G, et al. Systematic assessment of venous thromboembolism in COVID-19 patients receiving thromboprophylaxis: incidence and role of D-dimer as predictive factors. J Thromb Thrombolysis 2020;50(01):211-216

34 Bompard F, Monnier H, Saab I, et al. Pulmonary embolism in patients with COVID-19 pneumonia. Eur Respir J 2020;56(01):2001365

35 Chen S, Zhang D, Zheng T, Yu Y, Jiang J. DVT incidence and risk factors in critically ill patients with COVID-19. J Thromb Thrombolysis 2020. Doi: 10.1007/s11239-020-02181-w

36 Cho ES, McClelland PH, Cheng O, et al. Utility of D-dimer for diagnosis of deep vein thrombosis in coronavirus disease-19 infection. J Vasc Surg Venous Lymphat Disord 2021;9(01):47-53

37 Criel M, Falter M, Jaeken J, et al. Venous thromboembolism in SARS-CoV-2 patients: only a problem in ventilated ICU patients, or is there more to it? Eur Respir J 2020;56(01):2001201

38 Cui S, Chen S, Li X, Liu S, Wang F. Prevalence of venous thromboembolism in patients with severe novel coronavirus pneumonia.J Thromb Haemost 2020;18(06):1421-1424

39 Fauvel C, Weizman O, Trimaille A, et al;Critical Covid-19 France Investigators. Pulmonary embolism in COVID-19 patients: a French multicentre cohort study. Eur Heart J 2020;41(32): 3058-3068

40 Grandmaison G, Andrey A, Périard D, et al. Systematic screening for venous thromboembolic events in COVID-19 pneumonia. TH Open 2020;4(02):e113-e115

41 Koleilat I, Galen B, Choinski K, et al. Clinical characteristics of acute lower extremity deep venous thrombosis diagnosed by duplex in patients hospitalized for coronavirus disease 2019. J Vasc Surg Venous Lymphat Disord 2021;9(01):36-46

42 Llitjos J-F, Leclerc M, Chochois C, et al. High incidence of venous thromboembolic events in anticoagulated severe COVID-19 patients. J Thromb Haemost 2020;18(07):1743-1746

43 Lodigiani C, Iapichino G, Carenzo L, et al;Humanitas COVID-19 Task Force. Venous and arterial thromboembolic complications in COVID-19 patients admitted to an academic hospital in Milan, Italy. Thromb Res 2020;191:9-14

44 Mei F, Fan J, Yuan J, et al. Comparison of venous thromboembolism risks between COVID-19 pneumonia and community-acquired pneumonia patients. Arterioscler Thromb Vasc Biol 2020;40(09): 2332-2337

45 Middeldorp S, Coppens M, van Haaps TF, et al. Incidence of venous thromboembolism in hospitalized patients with COVID-19. J Thromb Haemost 2020;18(08):1995-2002

46 Moll M, Zon RL, Sylvester KW, et al. VTE in ICU patients with COVID-19. Chest 2020;158(05):P2130-P2135

47 Pesavento R, Ceccato D, Pasquetto G, et al. The hazard of (sub) therapeutic doses of anticoagulants in non-critically ill patients with Covid-19: the Padua province experience. J Thromb Haemost 2020. Doi: $10.1111 /$ jth. 15022

48 Ren B, Yan F, Deng Z, et al. Extremely high incidence of lower extremity deep venous thrombosis in 48 patients with severe COVID-19 in Wuhan. Circulation 2020;142(02):181-183

49 Santoliquido A, Porfidia A, Nesci A, et al;GEMELLI AGAINST COVID-19 Group. Incidence of deep vein thrombosis among non-ICU patients hospitalized for COVID-19 despite pharmacological thromboprophylaxis. J Thromb Haemost 2020;18(09): 2358-2363

50 Tavazzi G, Civardi L, Caneva L, Mongodi S, Mojoli F. Thrombotic events in SARS-CoV-2 patients: an urgent call for ultrasound screening. Intensive Care Med 2020;46(06):1121-1123

51 Thomas W, Varley J, Johnston A, et al. Thrombotic complications of patients admitted to intensive care with COVID-19 at a teaching hospital in the United Kingdom. Thromb Res 2020;191:76-77

52 Aleva FE, van Mourik L, Broeders MEAC, Paling AJ, de Jager CPC. COVID-19 in critically ill patients in North Brabant, the Netherlands: patient characteristics and outcomes. J Crit Care 2020;60:111-115

53 Dugar S, Duggal A, Bassel A, Soliman M, Moghekar A. Spontaneous echo contrast in venous ultrasound of severe COVID-19 patients. Intensive Care Med 2020;46(08):1637-1639

54 Hanif A, Khan S, Mantri N, et al. Thrombotic complications and anticoagulation in COVID-19 pneumonia: a New York City hospital experience. Ann Hematol 2020;99(10):2323-2328

55 Le Jeune S, Suhl J, Benainous R, et al. High prevalence of early asymptomatic venous thromboembolism in anticoagulated COVID-19 patients hospitalized in general wards. J Thromb Thrombolysis 2020. Doi: 10.1007/s11239-020-02246-w

56 Mattioli M, Benfaremo D, Mancini M, et al. Safety of intermediate dose of low molecular weight heparin in COVID-19 patients. J Thromb Thrombolysis 2020. Doi: 10.1007/s11239-020-02243-z

57 Paolisso P, Bergamaschi L, D’Angelo EC, et al. Preliminary experience with low molecular weight heparin strategy in COVID-19 patients. Front Pharmacol 2020;11(101548923):1124

58 Pizzolo F, Rigoni AM, De Marchi S, et al. Deep vein thrombosis in SARS-CoV-2 pneumonia-affected patients within standard care units: exploring a submerged portion of the iceberg. Thromb Res 2020;194:216-219

59 Nadkarni GN, Lala A, Bagiella E, et al. Anticoagulation, bleeding, mortality, and pathology in hospitalized patients with COVID-19. J Am Coll Cardiol 2020;76(16):1815-1826

60 Flumignan RLG, Tinôco JDdS, Pascoal PIF, et al. Prophylactic anticoagulants for people hospitalised with COVID-19. Cochrane Database Syst Rev 2020. Doi: 10.1002/14651858.CD013739

61 Kunutsor SK, Laukkanen JA. Incidence of venous and arterial thromboembolic complications in COVID-19: a systematic review and meta-analysis. Thromb Res 2020;196:27-30 
62 Rosovsky RP, Sanfilippo KM, Wang TF, et al. Anticoagulation practice patterns in COVID-19: a global survey. Res Pract Thromb Haemost 2020;4(06):969-983

63 Cohen AT, Spiro TE, Spyropoulos ACMAGELLAN Steering Committee. Rivaroxaban for thromboprophylaxis in acutely ill medical patients. N Engl J Med 2013;368(20):1945-1946

64 Leizorovicz A, Cohen AT, Turpie AG, Olsson CG, Vaitkus PT, Goldhaber SZPREVENT Medical Thromboprophylaxis Study Group. Randomized, placebo-controlled trial of dalteparin for the prevention of venous thromboembolism in acutely ill medical patients. Circulation 2004;110(07):874-879
65 Samama MM, Cohen AT, Darmon JY, et al;Prophylaxis in Medical Patients with Enoxaparin Study Group. A comparison of enoxaparin with placebo for the prevention of venous thromboembolism in acutely ill medical patients. N Engl J Med 1999;341(11):793-800

66 Cook D, Crowther M, Meade M, et al. Deep venous thrombosis in medical-surgical critically ill patients: prevalence, incidence, and risk factors. Crit Care Med 2005;33(07):1565-1571

67 Chi G, Lee JJ, Jamil A, et al. Venous thromboembolism among hospitalized patients with COVID-19 undergoing thromboprophylaxis: a systematic review and meta-analysis. J Clin Med 2020;9(08):E2489 